\title{
Parameter Estimation for Long-Memory Stochastic Volatility at Discrete Observation
}

\author{
Xiaohui Wang and Weiguo Zhang \\ School of Business Administration, South China University of Technology, Guangzhou, China \\ Correspondence should be addressed to Weiguo Zhang; wgzhang@scut.edu.cn
}

Received 12 December 2013; Accepted 26 February 2014; Published 25 March 2014

Academic Editor: Weilin Xiao

Copyright ( 2014 X. Wang and W. Zhang. This is an open access article distributed under the Creative Commons Attribution License, which permits unrestricted use, distribution, and reproduction in any medium, provided the original work is properly cited.

Ordinary least squares estimators of variogram parameters in long-memory stochastic volatility are studied in this paper. We use the discrete observations for practical purposes under the assumption that the Hurst parameter $H \in(1 / 2,1)$ is known. Based on the ordinary least squares method, we obtain both the explicit estimators for drift and diffusion by minimizing the distance function between the variogram and the data periodogram. Furthermore, the resulting estimators are shown to be consistent and to have the asymptotic normality. Numerical examples are also presented to illustrate the performance of our method.

\section{Introduction}

There has been a long history about the research of volatility modelling and estimation from historical data since the stochastic volatility was considered (see Taylor [1]). A series of studies found that the volatility of stock price often had long range dependence (LRD), meaning that the autocorrelations of volatility process decreased at a hyperbolic rate and were nonsummable (see Robinson [2]). Some work about the long-memory stochastic volatility (LMSV) has been done to describe and explain these slowly decaying autocorrelations. There were a wide variety of LMSV models, in terms of discrete and continuous time. Breidt et al. [3] and Harvey [4] independently considered an LMSV case where the log-volatility was modelled as a fractionally integrated ARMA (autoregressive, integrated, and moving average) or FARIMA process. Robinson and Zaffaroni [5] formulated a nonlinear time series model of long-memory stochastic volatility. Comte and Renault [6] and Comte et al. [7] proposed a continuous-time fractionally stochastic volatility (FSV) model which adopted a simpler representation of the fractional Brownian motion. Chronopoulou and Viens [8] did a comparison of how poor many longmemory-parameter estimators were for real data, using various nonparametric methods, and they proposed implied $H$ approach.

Correspondingly, the statistical inference problem of a volatility process under discrete and continuous cases is quite important in the implements of LMSV. In terms of the discrete-time models, Geweke and Porter-Hudak [9] proposed a log-periodogram regression method. Furthermore, the expressions for asymptotic bias and variance of the estimators were presented by Deo and Hurvich [10]. Arteche [11] developed parametric and semiparametric methods to estimate the memory parameters. Chen and Deo [12] specified and developed the generalized method of moments (GMM) in a linear long memory process. Brockwell and Davis [13] also showed frequency domain quasimaximum likelihood (FDQML) estimators that were used in long-memory stochastic volatility. However, very few papers developed the parameter estimations for long-memory stochastic volatility in the continuous-time case. Comte and Renault [6] used the log-periodogram regression approach to estimate the long-memory parameter. Their empirical study on CAC40 of the Paris Stock Exchange confirmed the short-memory feature of stock price but illustrated the long-memory feature of the volatility. Based on a first-order approximate version of the volatility process, Casas and Gao [14] estimated 
the parameters in a special class of LMSV models by Whittle estimation method. Chronopoulou and Viens $[8,15]$ used real S\&P 500 data to compare the numerical performance of the GPH estimator and the implied value of $H$ by calibrating it to option price. Their empirical study showed the instability of $\mathrm{GPH}$ estimator.

Perhaps, the most popular approach to modelling long memory in continuous time is to use fractional Brownian motion ( $\mathrm{fBm})$ as a long-memory driving source in the stochastic volatility. We assume that the stochastic volatility follows a fractional Ornstein-Uhlenbeck process (fOU, see Cheridito et al. [16], Xiao et al. [17], and Hu and Nualart [18]). It is worthwhile to mention that it is usually only possible to observe asset price at points of discrete time. Therefore, statistical inference of long-memory stochastic volatility through discretely observed asset price is of great interest for practical purposes. It would be optimal to estimate the parameters of fractional Ornstein-Uhlenbeck process and the memory-parameter $H$ jointly. However, in the majority of the long-memory models, a rigorous way to jointly estimate this vector of parameters is unknown. Rosenbaum [19] provides a strongly consistent estimator of $H$ for unobserved stochastic volatility, and he proves that the convergence speed is very slow, even though his estimator reaches optimal speed. His research indicates another reason why one should try to select $H$ using calibration instead of estimation. Chronopoulou and Viens [8, 15] suggest to estimate the parameters separately. They propose using a calibration technique to fit models with various Hurst indexes to the options market, picking the model with the best $H$. In this paper, we consider the estimators of the variogram parameters from discrete observations through the ordinary least squares method under the assumption that $H$ is known. Here, the main obstacles are the fact that volatility itself is never directly observed and variogram method is limited to stationary process. In this paper, we refer to Viens [20] for the idea of filter in the conditional probability distribution of stochastic volatility through the discrete observations of asset price. In addition, we also refer to Cheridito et al. [16] for the stationary version solution of fractional Ornstein-Uhlenbeck process and the asymptotic form of autocovariance function when the time lag goes to infinity.

This paper contributes to the expositions on theoretical and practical aspects of parameter estimation for longmemory stochastic volatility which follows a fractional Ornstein-Uhlenbeck process. Firstly, we obtain the variance of the stationary version solution to the fractional OrnsteinUhlenbeck process, which is important in identifying the variogram function. Then, we deduce the approximate form of the ordinary least squares estimators of $(\alpha, \beta)$ in longmemory stochastic volatility. Finally, the asymptotic properties are given in this paper.

The remainder of this paper proceeds as follows. In Section 2, we state the estimation procedure. In Section 3, we prove the asymptotic properties of the estimators. In Section 4, we give simulation examples to show the performance of these estimators. Concluding remarks are given in Section 5 .

\section{The Method of Estimation at Discrete Observation}

Let $\left(\Omega, \mathscr{F},\left(\mathscr{F}_{t}\right)_{t \geq 0}, P\right)$ be a complete probability space on which a standard Brownian motion $W_{t}$ and a fractional Brownian motion $B_{t}^{H}$ with Hurst exponent $H \in(1 / 2,1)$ are well defined, and $\mathscr{F}_{t}=\sigma\left\{W_{\tau}, B_{\tau}^{H}, 0 \leq \tau \leq t\right\}$. We assume that the asset price process $\left\{X_{t} ; t \geq 0\right\}$ is conditionally lognormal and the volatility process $\left\{Y_{t} ; t \geq 0\right\}$ is a fractional OrnsteinUhlenbeck process. $\left\{X_{t} ; t \geq 0\right\}$ and $\left\{Y_{t} ; t \geq 0\right\}$ satisfy the following equations:

$$
\frac{d X_{t}}{X_{t}}=\sigma\left(Y_{t}\right) d W_{t}, \quad d Y_{t}=-\alpha Y_{t} d t+\beta d B_{t}^{H},
$$

where $\alpha$ is the rate of reversion, $\beta$ is the volatility of the volatility process, $(\alpha, \beta) \in \Theta \subset \mathbb{R}^{2}$, and $\Theta$ is compact. $\sigma(\cdot)$ is a chosen deterministic function. Here, we choose Scott [21] model to make sure that the volatility remains positive; that is, $\sigma(y)=e^{y}$. In this paper, we also assume that $\left\{W_{t} ; t \geq 0\right\}$ is independent of $\left\{B_{t}^{H} ; t \geq 0\right\}$. Since there are no leverage effects in our model, thus the model is identical whether one uses a signed function $\sigma(y)$ or its absolute value.

Before discussing the problem in this paper, we need the following results taken from Cheridito et al. [16]. The stationary version solution to (1) admits the following representation:

$$
Y_{t}=\beta \int_{-\infty}^{t} e^{-\alpha(t-s)} d B_{s}^{H}
$$

with initial condition $Y_{0}=\beta \int_{-\infty}^{0} e^{\alpha s} d B_{s}^{H}$ which is explicitly contained in the representation (2). The asymptotic autocovariance of $\left\{Y_{t} ; t \geq 0\right\}$ is given in Theorem 2.3 in Cheridito et al. [16] as

$$
\begin{aligned}
\operatorname{Cov}\left(Y_{t}, Y_{t+s}\right)= & \frac{1}{2} \beta^{2} \sum_{n=1}^{N} \alpha^{-2 n}\left(\prod_{k=0}^{2 n-1}(2 H-k)\right) s^{2 H-2 n} \\
& +O\left(s^{2 H-2 N-2}\right)
\end{aligned}
$$

for fixed $t \in \mathbb{R}, N=1,2, \ldots$, and $s \rightarrow \infty$.

The following lemma, whose proof is given in Appendix, gives the variance of $\left\{Y_{t} ; t \geq 0\right\}$ which is important for the identification of the variogram function.

Lemma 1. Let $1 / 2<H<1$; then $\left\{Y_{t} ; t \geq 0\right\}$ is a Gaussian process with $E\left[Y_{t}\right]=0$ and $\operatorname{Var}\left[Y_{t}\right]=\Gamma(2 H) H \alpha^{-2 H} \beta^{2}$ for every $t \in \mathbb{R}$.

Now, suppose that the price process is observed at the discrete time instants $\left(t_{1}, t_{2}, \ldots, t_{N+1}\right)$ and $t_{i}=i \Delta t$, $i=1,2, \ldots, N+1$, for some fixed length $\Delta t>0$. Use $\left(X_{1}, \ldots, X_{N+1}\right)$ and $\left(Y_{1}, \ldots, Y_{N+1}\right)$ to represent $\left(X_{t_{1}}\right.$, $\left.\ldots, X_{t_{N+1}}\right)$ and $\left(Y_{t_{1}}, \ldots, Y_{t_{N+1}}\right)$, respectively. Nourdin [22] and Neuenkirch and Nourdin [23] give the error of Euler approximations for the homogeneous one-dimensional SDEs involving $\mathrm{fBm}$ and having bounded coefficients. Mishura [24] presents the rate of convergence of Euler approximations of 
the solutions for SDE with nonstationary coefficient. Inspired by their work, we consider the Euler discretization in the form of

$$
\begin{gathered}
\Delta Y_{i}:=Y_{i+1}-Y_{i}=-\alpha Y_{i} \Delta t+\beta\left(B_{t_{i+1}^{H}}^{H}-B_{t_{i}}^{H}\right), \\
\Delta X_{i}:=X_{i+1}-X_{i}=X_{i} e^{Y_{i}} \xi_{i} \sqrt{\Delta t},
\end{gathered}
$$

where $\left\{\xi_{i}\right\}$ and $\left\{B_{i}^{H}\right\}$ are independent sequences and $\left\{\xi_{i}\right\}$ is an independent sequence of $N(0,1)$ random variables.

For simplicity, we denote

$$
\mu=\beta^{2} H \alpha^{-2 H} \text {. }
$$

In what follows, it is necessary to estimate the parameters $(\alpha, \mu)$ first. We still denote $(\alpha, \mu) \in \Theta$ below where $\Theta$ is compact. Then, we will get the estimators of $(\alpha, \beta)$ when $H \in$ $(1 / 2,1)$ is known.

Volatility itself cannot be directly observed. In practice, even for the most liquid assets or indexes, one would be restricted to using the values of the asset at discrete times. Therefore, we normalize the fluctuation sequence

$$
D_{i}=\frac{\Delta X_{i}}{X_{i} \sqrt{\Delta t}}
$$

which is proved to be the discrete observed realization of $e^{Y_{i}} \xi_{i}$ in Lemmas 2 and 3.

Lemma 2. Let $\tau \ll 1$ and $M_{t}=\int_{0}^{t} e^{Y_{s}} d W_{s}$. Then,

$$
E\left[\ln X_{t+\tau}-\ln X_{t}\right]^{2}=E\left[M_{t+\tau}-M_{t}\right]^{2}+O(\tau) .
$$

Proof of Lemma 2. Since $\left\{W_{t} ; t \geq 0\right\}$ is independent of $\left\{B_{t}^{H} ; t \geq 0\right\}$, so we have

$$
\begin{gathered}
X_{t}=e^{X_{0}} e^{-\int_{0}^{t} e^{2 Y_{s}} d s+\int_{0}^{t} e^{Y_{s}} d W_{s},} \\
X_{t+\tau}=e^{X_{0}} e^{-\int_{0}^{t+\tau} e^{2 Y_{s}} d s+\int_{0}^{t+\tau} e^{Y_{s}} d W_{s} .}
\end{gathered}
$$

Thus,

$$
\ln \frac{X_{t+\tau}}{X_{t}}=-\int_{t}^{t+\tau} e^{2 Y_{s}} d s+\int_{t}^{t+\tau} e^{Y_{s}} d W_{s}
$$

where $\int_{t}^{t+\tau} e^{Y_{s}} d W_{s}$ is the martingale term of $M_{t+\tau}-M_{t}$.

According to Belfadli et al. [25], for all $\varepsilon \in(0, H), Y_{t}$ admits a modification with $(H-\varepsilon)$-Hölder continuous paths. Replace $e^{Y_{s}}$ by $e^{Y_{t}}$ at the order $O(\tau)$. Then,

$$
\begin{aligned}
E\left[\left(\ln \frac{X_{t+\tau}}{X_{t}}\right)^{2}\right] & =E\left[\left(-e^{2 Y_{t}} \tau+\int_{t}^{t+\tau} e^{Y_{s}} d W_{s}\right)^{2}\right] \\
& =E\left[e^{2 Y_{t}}\right] \tau+O(\tau)
\end{aligned}
$$

Therefore, $E\left[\ln X_{t+\tau}-\ln X_{t}\right]^{2}$ and $E\left[M_{t+\tau}-M_{t}\right]^{2}$ are equivalent at the order $O(\tau)$. Additionally,

$$
E\left[M_{t+\tau}-M_{t}\right]^{2}=\int_{t}^{t+\tau} E\left[e^{2 Y_{s}}\right] d s=\tau e^{2 \beta^{2} H \alpha^{-2 H} \Gamma(2 H)} .
$$

Thus, this completes the proof.
Lemma 3. Let $\tau \ll 1, t_{1}+\tau<t_{2}, M_{t}=\int_{0}^{t} e^{Y_{s}} d W_{s}$. Then,

$$
\begin{aligned}
E & {\left[\left(\ln X_{t_{1}+\tau}-\ln X_{t_{1}}\right)^{2}\left(\ln X_{t_{2}+\tau}-\ln X_{t_{2}}\right)^{2}\right] } \\
& =E\left[\left(M_{t_{1}+\tau}-M_{t_{1}}\right)^{2}\left(M_{t_{2}+\tau}-M_{t_{2}}\right)^{2}\right]+O\left(\tau^{2}\right) .
\end{aligned}
$$

Proof of Lemma 3. Since $\left\{Y_{t} ; t \geq 0\right\}$ and $\left\{W_{t} ; t \geq 0\right\}$ are independent, we replace $e^{Y_{s}}$ by $e^{Y_{t_{1}}}$ and $e^{Y_{u}}$ by $e^{Y_{t_{2}}}$ at the order $O(\tau)$, respectively; it follows that

$$
\begin{gathered}
E\left[\left(\ln X_{t_{1}+\tau}-\ln X_{t_{1}}\right)^{2}\left(\ln X_{t_{2}+\tau}-\ln X_{t_{2}}\right)^{2}\right] \\
=E\left[\left(-\int_{t_{1}}^{t_{1}+\tau} e^{2 Y_{s}} d s+\int_{t_{1}}^{t_{1}+\tau} e^{Y_{s}} d W_{s}\right)^{2}\right. \\
\left.\times\left(-\int_{t_{2}}^{t_{2}+\tau} e^{2 Y_{u}} d u+\int_{t_{2}}^{t_{2}+\tau} e^{Y_{u}} d W_{u}\right)^{2}\right] \\
=E\left[\left(\left(\int_{t_{1}}^{t_{1}+\tau} e^{Y_{s}} d W_{s}\right)^{2}+\left(\int_{t_{1}}^{t_{1}+\tau} e^{2 Y_{s}} d s\right)^{2}\right.\right. \\
\left.-2 \int_{t_{1}}^{t_{1}+\tau} e^{2 Y_{s}} d s \int_{t_{1}}^{t_{1}+\tau} e^{Y_{s}} d W_{s}\right)^{2} \\
\times\left(\left(\int_{t_{2}}^{t_{2}+\tau} e^{Y_{u}} d W_{u}\right)^{2}+\left(\int_{t_{2}}^{t_{2}+\tau} e^{2 Y_{u}} d u\right)^{2}\right. \\
\left.\left.-2 \int_{t_{2}}^{t_{2}+\tau} e^{2 Y_{u}} d u \int_{t_{2}}^{t_{2}+\tau} e^{Y_{u}} d W_{u}\right)\right] \\
=E\left[\left(\int_{t_{1}}^{t_{1}+\tau} e^{Y_{s}} d W_{s}\right)^{2}\left(\int_{t_{2}}^{t_{2}+\tau} e^{Y_{u}} d W_{u}\right)^{2}\right]+O\left(\tau^{2}\right),
\end{gathered}
$$

which completed the proof of Lemma 3.

Applying Lemmas 2 and 3, we can get the discrete observations of volatility through asset price.

Let

$$
L_{t}=\ln \left|D_{t}\right|
$$

Define the variogram function $v(t, \alpha, v)$ as half the variance of the increments of $L_{t}$ :

$$
2 v(t, \alpha, \mu)=E\left[\left(L_{t+s}-L_{s}\right)^{2}\right] .
$$

For each fixed $H \in(1 / 2,1)$, we consider an ordinary least squares variogram (see Lahiri et al. [26]) to obtain the estimators of $(\alpha, \mu)$ by minimizing a certain distance between the variogram and the data periodogram at a finite number of lags. The ordinary least squares method of estimating variogram parameters used in this paper first needs to get the variogram function.

Let

$$
\gamma(t, \alpha, \mu)=\mu \Gamma(2 H)-\mu(2 H-1)(\alpha t)^{2 H-2} .
$$


For fractional Ornstein-Uhlenbeck process, it is not possible to get the exact variogram function. In Lemma 4, we give conditions on the time lag that ensure that $\gamma(t, \alpha, \mu)$ is an approximation of variogram function $v(t, \alpha, \mu)$ with an approximation error less than any $\varepsilon$.

Lemma 4. For any $\varepsilon>0$, there are some $K=9(2 H-2)(2 H-$ 3) and $T=(\varepsilon / K)^{1 /(2 H-4)}$, such that, for any $t>T$,

$$
|v(t, \alpha, \mu)-\gamma(t, \alpha, \mu)|<\varepsilon .
$$

Proof of Lemma 4. Applying Theorem 2.3 in [16], for any $\varepsilon>$ 0 , there exist $K=9(2 H-2)(2 H-3)$ and $T=(\varepsilon / K)^{1 /(2 H-4)}$, such that, for any $t>T$,

$$
\begin{gathered}
\left|\operatorname{cov}\left(Y_{0}, Y_{t}\right)-\mu(2 H-1)(\alpha t)^{2 H-2}\right| \\
\leq \mid(2 H-2)(2 H-3) t^{2 H-4} \\
\quad+2^{4-2 H}(2 H-2)(2 H-3) t^{2 H-4} \mid \\
<9(2 H-2)(2 H-3) t^{2 H-4}<\varepsilon .
\end{gathered}
$$

From Lemma 1 , it follows that, for any $\varepsilon>0$, if $t>T$, we have

$$
\begin{aligned}
\mid v & (t, \alpha, v)-\gamma(t, \alpha, \mu) \mid \\
& =\left|\frac{1}{2} E\left[\left(Y_{t}+\ln \left|\xi_{t}\right|-Y_{0}-\ln \left|\xi_{0}\right|\right)^{2}\right]-\gamma(t, \alpha, \mu)\right| \\
& =\left|E\left[Y_{0}^{2}\right]-E\left[Y_{0} Y_{t}\right]-\mu \Gamma(2 H)+\mu(2 H-1)(\alpha t)^{2 H-2}\right| \\
& =\left|\operatorname{cov}\left(Y_{0}, Y_{t}\right)-\mu(2 H-1)(\alpha t)^{2 H-2}\right|<\varepsilon .
\end{aligned}
$$

This completes the proof.

Accordingly, from (15), we have $\left\{L_{i \Delta t}\right\}_{i=1, \ldots, N}$ at the discrete time instants. For notational simplicity, when there is no chance of confusion, we will drop $\Delta t$ from the subscript $\left\{L_{i \Delta t}\right\}$ and write them as $\left\{L_{i}\right\}$. Let $\left\{h_{1} \Delta t, h_{2} \Delta t, \ldots, h_{M} \Delta t\right\}$ be a finite set of lag vectors. We denote $v\left(h_{k} \Delta t, \alpha, \mu\right)$ and $\gamma\left(h_{k} \Delta t, \alpha, \mu\right)$ by $v(k, \alpha, \mu)$ and $\gamma(k, \alpha, \mu)$, respectively, where $k=1,2, \ldots, M$.

In this paper, our results will require the following assumption for time lag in the parameter estimation problems to be satisfied.

Assumption 5. Consider (1) $\varepsilon \rightarrow 0$, (2) $h_{k} \Delta t>T, k=$ $1,2, \ldots, M$, where $K=9(2 H-2)(2 H-3)$ and $T=$ $(\varepsilon / K)^{1 /(2 H-4)}$, and $(3) H \in(1 / 2,1)$ and $H$ is known.

Ordinary least squares (OLS) estimate method for variogram parameters fits a parametric model of valid variograms to a pointwise nonparametric variogram estimator by minimizing their certain distance at a finite number of lags. Suppose that $W_{k, N}$ is a variogram estimator based on a sample of size $N$ and suppose that $2 v(k, \alpha, \mu)$ is a valid variogram model. The most ordinary choice of distance function is given by

$$
\sum_{k=1}^{M}\left[W_{k, N}-2 v(k, \alpha, \mu)\right]^{2}
$$

which, when minimized with respect to $\alpha$ and $\mu$, yields the ordinary least squares (OLS) estimators of $(\alpha, \mu)$. Therefore, the OLS estimation of variogram parameters $\alpha$ and $\mu$ is defined as

$$
(\widehat{\alpha}, \widehat{\mu})=\arg \min \left\{g_{N}(\alpha, \mu)^{\prime} g_{N}(\alpha, \mu)\right\},
$$

where

$$
g_{N}(\alpha, \mu)=\left(W_{1, N}-2 v(1, \alpha, \mu), \ldots, W_{M, N}-2 v(M, \alpha, \mu)\right)^{\prime} .
$$

In the following, we take $W_{k, N}$ as the moment estimator of $2 v(k, \alpha, v)$, which follows

$$
W_{k, N}=\frac{1}{N-h_{k}} \sum_{i=1}^{N-h_{k}}\left(L_{i+h_{k}}-L_{i}\right)^{2}, \quad k=1,2, \ldots, M .
$$

The main result is summarized in the following theorem.

Theorem 6. Let Assumption 5 be satisfied. Then,

$$
\begin{gathered}
\widehat{\alpha}=\left[\frac{2 M \widehat{\mu} \Gamma(2 H)-\sum_{k=1}^{M} W_{k, N}}{2 \widehat{\mu}(2 H-1) \sum_{k=1}^{M}\left(h_{k} \Delta t\right)^{2 H-2}}\right]^{1 /(2 H-2)}, \\
\widehat{\mu}=\left(\sum_{k=1}^{M} W_{k, N} \sum_{k=1}^{M}\left(h_{k} \Delta t\right)^{4 H-4}\right. \\
\left.-\sum_{k=1}^{M}\left(h_{k} \Delta t\right)^{2 H-2} \sum_{k=1}^{M} W_{k, N}\left(h_{k} \Delta t\right)^{2 H-2}\right) \\
\times\left(2 \Gamma(2 H)\left[M \sum_{k=1}^{M}\left(h_{k} \Delta t\right)^{4 H-4}-\left[\sum_{k=1}^{M}\left(h_{k} \Delta t\right)^{2 H-2}\right]^{2}\right]\right)^{-1} .
\end{gathered}
$$

Proof of Theorem 6. Let $g_{N}^{*}(\alpha, \mu)=\left(W_{1, N}-2 \gamma(1, \alpha, \mu), \ldots\right.$, $\left.W_{M, N}-2 \gamma(M, \alpha, \mu)\right)^{\prime}$. Since $W_{k, N}$ is the moment estimator of $v(k, \alpha, \mu)$, from Lemma 4 , it follows that

$$
\begin{gathered}
E\left[\left|g_{N}(\alpha, \mu)^{\prime} g_{N}(\alpha, \mu)-g_{N}^{*}(\alpha, \mu)^{\prime} g_{N}^{*}(\alpha, \mu)\right|\right] \\
=E\left[\mid 2 \sum_{k=1}^{M}\left[W_{k, N}-2 v(k, \alpha, \mu)\right]\right. \\
\times[2 \gamma(k, \alpha, \mu)-2 v(k, \alpha, \mu)] \\
\left.+\sum_{k=1}^{M}[2 \gamma(k, \alpha, \mu)-2 v(k, \alpha, \mu)]^{2} \mid\right]<\varepsilon .
\end{gathered}
$$

Then, $(\widehat{\alpha}, \widehat{\mu})$ follows that

$$
\begin{gathered}
\left.\frac{\partial\left(g_{N}^{*}(\alpha, \mu)^{\prime} g_{N}^{*}(\alpha, \mu)\right)}{\partial \alpha}\right|_{\alpha=\widehat{\alpha}}=0, \\
\left.\frac{\partial\left(g_{N}^{*}(\alpha, \mu)^{\prime} g_{N}^{*}(\alpha, \mu)\right)}{\partial \mu}\right|_{\mu=\widehat{\mu}}=0 .
\end{gathered}
$$


Substituting $g_{N}^{*}(\alpha, \mu)$ by its explicit expression, we have

$$
\begin{aligned}
& \sum_{k=1}^{M}\left[2 \widehat{\mu} \Gamma(2 H)-2 \widehat{\mu}(2 H-1)\left(\widehat{\alpha} h_{k} \Delta t\right)^{2 H-2}-W_{k, N}\right]=0, \\
& \sum_{k=1}^{M}\left[2 \widehat{\mu} \Gamma(2 H)-2 \widehat{\mu}(2 H-1)\left(\widehat{\alpha} h_{k} \Delta t\right)^{2 H-2}-W_{k, N}\right] \\
& \quad \times\left(h_{k} \Delta t\right)^{2 H-2}=0 .
\end{aligned}
$$

Then, (28) can be written as

$$
\begin{aligned}
& 2 M \widehat{\mu} \Gamma(2 H)-2 \widehat{\mu}(2 H-1) \widehat{\alpha}^{2 H-2} \sum_{k=1}^{M}\left(h_{k} \Delta t\right)^{2 H-2} \\
& -\sum_{k=1}^{M} W_{k, N}=0, \\
& 2 \widehat{\mu} \Gamma(2 H) \sum_{k=1}^{M}\left(h_{k} \Delta t\right)^{2 H-2} \\
& -2 \widehat{\mu}(2 H-1) \widehat{\alpha}^{2 H-2} \sum_{k=1}^{M}\left(h_{k} \Delta t\right)^{4 H-4} \\
& -\sum_{k=1}^{M} W_{k, N}\left(h_{k} \Delta t\right)^{2 H-2}=0 .
\end{aligned}
$$

Removing the common term $2 \widehat{\mu}(2 H-1) \widehat{\alpha}^{2 H-2}$ gives

$$
\begin{aligned}
& \frac{2 M \widehat{\mu} \Gamma(2 H)-\sum_{k=1}^{M} W_{k, N}}{\sum_{k=1}^{M}\left(h_{k} \Delta t\right)^{2 H-2}} \\
& =\frac{2 \widehat{\mu} \Gamma(2 H) \sum_{k=1}^{M}\left(h_{k} \Delta t\right)^{2 H-2}-\sum_{k=1}^{M} W_{k, N}\left(h_{k} \Delta t\right)^{2 H-2}}{\sum_{k=1}^{M}\left(h_{k} \Delta t\right)^{4 H-4}} .
\end{aligned}
$$

Solving for $\widehat{\mu}$ in (30) gives

$$
\begin{aligned}
\widehat{\mu}=\left(\sum_{k=1}^{M} W_{k, N} \sum_{k=1}^{M}\left(h_{k} \Delta t\right)^{4 H-4}\right. \\
\left.\quad-\sum_{k=1}^{M}\left(h_{k} \Delta t\right)^{2 H-2} \sum_{k=1}^{M} W_{k, N}\left(h_{k} \Delta t\right)^{2 H-2}\right) \\
\quad \times\left(2 \Gamma(2 H)\left[M \sum_{k=1}^{M}\left(h_{k} \Delta t\right)^{4 H-4}-\left[\sum_{k=1}^{M}\left(h_{k} \Delta t\right)^{2 H-2}\right]^{2}\right]\right)^{-1} .
\end{aligned}
$$

Substituting for $\widehat{\mu}$ in (28) gives

$$
\widehat{\alpha}=\left[\frac{2 M \widehat{\mu} \Gamma(2 H)-\sum_{k=1}^{M} W_{k, N}}{2 \widehat{\mu}(2 H-1) \sum_{k=1}^{M}\left(h_{k} \Delta t\right)^{2 H-2}}\right]^{1 /(2 H-2)} .
$$

Thus, the proof is completed.
Remark 7. According to the estimators of parameters $(\alpha, \mu)$ and (6), we can easily get the representation of $\widehat{\beta}$ as $\widehat{\beta}=$ $\sqrt{\widehat{\mu} \widehat{\alpha}^{2 H} / H}$.

\section{The Asymptotic Properties of the Estimators}

Theorem 8. Let Assumption 5 be satisfied; then the estimators $(\widehat{\alpha}, \widehat{\mu})$ converge to $(\alpha, \mu)$ with probability 1 as $N$ goes to infinity.

Proof of Theorem 8. Theorem 3.1 in Lahiri et al. [26] gives the sufficient conditions for that OLS estimators are consistent as follows.
(C1) For any $\left(\alpha_{1}, \mu_{1}\right),\left(\alpha_{2}, \mu_{2}\right) \in \Theta, \sum_{k=1}^{M}\left(2 \gamma\left(k ; \alpha_{1}, \mu_{1}\right)\right.$ $\left.-2 \gamma\left(k ; \alpha_{2}, \mu_{2}\right)\right)^{2} \geq 0$.
(C2) $\sup \{\gamma(k ; \alpha, \mu), h \in R,(\alpha, \mu) \in \Theta\}<\infty$, and $\gamma(k ; \alpha, \mu)$ has continuous partial derivatives of order $s(\geq 0)$ with respect to $\alpha$ and $\mu$.

(C3) For any $k=1,2, \ldots, M, W_{k, N}$ is consistent for $2 v(k ; \theta)$.

Therefore, it remains to check the conditions required to complete this proof.

Since $\Theta$ is compact, it is obvious that, for any $\left(\alpha_{1}, \mu_{1}\right),\left(\alpha_{2}, \mu_{2}\right) \in \Theta$, we have

$$
\sum_{k=1}^{M}\left(\gamma\left(k, \alpha_{1}, \mu_{1}\right)-\gamma\left(k, \alpha_{2}, \mu_{2}\right)\right)^{2} \geq 0
$$

Then, it is not difficult to show that $\gamma(k, \alpha, \mu)$ is continuous with respect to $\alpha$ and $\mu$ as well as $\sup \{\gamma(k, \alpha, \mu): \alpha, \mu \in \Theta\}<$ $\infty$ when $h_{k}>T$, for any $k=1,2, \ldots, M$.

According to Lemma 4, for any $\varepsilon>0$, if $h_{k} \Delta t>T, k=$ $1,2, \ldots M$, we have

$$
|2 v(k, \alpha, \mu)-2 \gamma(k, \alpha, \mu)|<2 \varepsilon
$$

Since $W_{k, N}$ is the moment estimator of $2 v(k, \alpha, \mu)$, using the consistent results of Hall [28], for any $\varepsilon>0$, then we get

$$
\begin{aligned}
& P\left\{\left|W_{k, N}-2 \gamma(k, \alpha, \mu)\right|>3 \varepsilon\right\} \\
& \leq P\left\{\left|W_{k, N}-2 v(k, \alpha, \mu)\right|\right. \\
& \quad+|2 v(k, \alpha, \mu)-2 \gamma(k, \alpha, \mu)|>3 \varepsilon\} \stackrel{N \rightarrow \infty}{\longrightarrow} 0 .
\end{aligned}
$$

Therefore, conditions (C1)-(C3) hold for our estimators. It is sufficient to deduce the desired result.

Next, we consider the distribution of the ordinary least squares estimators. We refer to Lahiri et al. [26] for the detailed notations. For any lag $\left(h_{1}, h_{2}, \ldots, h_{M}\right)$, let $g_{j}(\alpha, \mu)$ be an $M \times 1$ vector of partial derivatives of 
$(\gamma(1, \alpha, \mu), \gamma(2, \alpha, \mu), \ldots, \gamma(M, \alpha, \mu))$ with the $j$ th parameter; that is,

$$
\begin{aligned}
& g_{1}(\alpha, \mu) \\
& =\left(\frac{\partial}{\partial \alpha} \gamma(1, \alpha, \mu), \frac{\partial}{\partial \alpha} \gamma(2, \alpha, \mu), \ldots, \frac{\partial}{\partial \alpha} \gamma(M, \alpha, \mu)\right)^{\prime}, \\
& g_{2}(\alpha, \mu) \\
& =\left(\frac{\partial}{\partial \mu} \gamma(1, \alpha, \mu), \frac{\partial}{\partial \mu} \gamma(2, \alpha, \mu), \ldots, \frac{\partial}{\partial \mu} \gamma(M, \alpha, \mu)\right)^{\prime} .
\end{aligned}
$$

Also, set $\Gamma(\alpha, \mu)=\left(-2 g_{1}(\alpha, \mu),-2 g_{2}(\alpha, \mu)\right)$ to be a matrix of dimension $M \times 2$.

As derived in Theorem 3.2 in Lahiri et al. [26], if the nonparametric variogram estimator $W_{k, N}$ is asymptotically normal, then the OLS estimators of $(\alpha, \mu)$ are asymptotically normal with the same scaling constant that yield the asymptotic distribution for $W_{k, N}$. Therefore, we need to prove the asymptotically normal property of $W_{k, N}$ first. The asymptotic normality of $(\widehat{\alpha}, \widehat{\mu})$ is presented as follows.

Theorem 9. Let Assumption 5 be satisfied; then one has the following results:

(1)

$$
\sqrt{N}\left(\left(W_{1, N}-2 \gamma_{1}\right), \ldots,\left(W_{M, N}-2 \gamma_{M}\right)\right)^{\prime} \stackrel{d}{\longrightarrow} N\left(0, \Sigma_{1}(\alpha, \mu)\right)
$$

as $N \rightarrow \infty$, where the $(i, j)$ th element of $\Sigma_{1}(\alpha, \mu)$ is given by

$$
\left(\Sigma_{1}(\alpha, \mu)\right)_{i, j}=\Sigma_{s \in N} \operatorname{cov}\left(\left(L_{0}-L_{h_{i}}\right)^{2},\left(L_{s}-L_{s+h_{j}}\right)^{2}\right) ;
$$

(2)

$$
\sqrt{N}((\widehat{\alpha}, \widehat{\mu})-(\alpha, \mu))^{\prime} \stackrel{d}{\longrightarrow} N\left(0, \Sigma_{V}(\alpha, \mu)\right)
$$

as $N \rightarrow \infty$, where

$$
\begin{gathered}
\Sigma_{V}(\alpha, \mu)=B(\alpha, \mu) \Gamma^{\prime}(\alpha, \mu) \Sigma_{1}(\alpha, \mu) \Gamma(\alpha, \mu) B(\alpha, \mu), \\
B(\alpha, \mu)=\left(\Gamma(\alpha, \mu)^{\prime} \Gamma(\alpha, \mu)\right)^{-1} .
\end{gathered}
$$

Proof of Theorem 9. In order to prove (37), by the CramerWold device, it is enough to show that, for any $\mathbf{a}=\left(a_{1}, a_{2}, \ldots\right.$, $\left.a_{M}\right)^{\prime} \in R^{M}$, and $\sum_{k=1}^{M}\left|a_{k}\right|<\infty, F_{1 n}=\sum_{k=1}^{M} \sqrt{N} a_{k}\left(W_{k, N}-\right.$ $2 \gamma(k, \alpha, \mu)) \stackrel{d}{\rightarrow} N\left(0, \mathbf{a}^{\prime} \Sigma_{1}(\alpha, \mu) \mathbf{a}\right)$. Fix $\mathbf{a} \in R^{M}$ and let $\varepsilon=1 / N$ and Assumption 5 hold; then define

$$
\begin{aligned}
& F_{2 n}=\sum_{k=1}^{M} \sqrt{N} a_{k}\left\{|N|^{-1} \sum_{i=1}^{N}\left(L_{i}-L_{i+h_{k}}\right)^{2}-2 \gamma(k, \alpha, \mu)\right\}, \\
& F_{3 n}=\sum_{k=1}^{M} \sqrt{N} a_{k}\left\{|N|^{-1} \sum_{i=1}^{N}\left(L_{i}-L_{i+h_{k}}\right)^{2}-2 v(k, \alpha, \mu)\right\} .
\end{aligned}
$$

Based on Cauchy-Schwarz theorem and Lemma A.1 in Lahiri et al. [26], there exist $C_{1}$ and $C_{2}$ satisfying the following inequations:

$$
\begin{aligned}
& E\left|F_{1 n}-F_{2 n}\right| \\
& \leq \sqrt{N} \sum_{k=1}^{N}\left|a_{k}\right|\left[\left|\left(N-h_{k}\right)^{-1}-N^{-1}\right| E\left|\sum_{i=1}^{N-h_{k}}\left(L_{i}-L_{i+h_{k}}\right)^{2}\right|\right. \\
& \left.+N^{-1} E\left|\sum_{i=N-h_{k}+1}^{N}\left(L_{i}-L_{i+h_{k}}\right)^{2}\right|\right] \\
& \leq C_{1} \sqrt{N} \sum_{k=1}^{N}\left|a_{k}\right|\left[|| N-h_{k}|-N| N^{-2}\right. \\
& \times\left(E\left(\sum_{i=1}^{N}\left(L_{i}-L_{i+h_{k}}\right)^{2}\right)^{2}\right)^{1 / 2}+N^{-1} \\
& \times\left\{\sum_{s \in N} h_{k} \mid \operatorname{cov}\left(\left(L_{0}-L_{h_{k}}\right)^{2},\right.\right. \\
& \left.\left.\left.\left(L_{s}-L_{s+h_{k}}\right)^{2}\right) \mid\right\}^{1 / 2}\right] \\
& \leq C_{2} \sum_{k=1}^{N}\left|a_{k}\right|\left[N^{-1}|N-| N-h_{k}||+N^{-1 / 2} h_{k}^{1 / 2}\right] \\
& \longrightarrow 0 \text { as } N \longrightarrow \infty \text {. }
\end{aligned}
$$

Then, from Lemma 4, we have

$$
\begin{aligned}
\left|F_{2 n}-F_{3 n}\right| & \leq \sqrt{N} \sum_{k=1}^{N}\left|a_{k}\right||2 v(k, \alpha, \mu)-2 \gamma(k, \alpha, \mu)| \\
& \leq 2 N^{1 / 2} N^{-1} \sum_{k=1}^{N}\left|a_{k}\right| \stackrel{N \rightarrow \infty}{\longrightarrow} 0 .
\end{aligned}
$$

By Lemma A.1 in Lahiri et al. [26], we could obtain that, as $N \rightarrow \infty$,

$$
F_{3 n} \stackrel{d}{\longrightarrow} N\left(0, \mathbf{a}^{\prime} \Sigma_{1}(\alpha, \mu) \mathbf{a}\right)
$$

This implies that $F_{1 n}$ converges to $N\left(0, \mathbf{a}^{\prime} \Sigma_{1}(\alpha, \mu) \mathbf{a}\right)$ in distribution as $N$ goes to infinity. Thus, we complete the proof of (37).

Finally, (37) combined with conditions (C1)-(C3) verified in Theorem 8, we conclude the desired result in (39).

\section{Numerical Illustration}

In this section, we present numerical examples for different values of $H, \alpha$, and $\beta$ to illustrate the performance of our 
TABLE 1: The means and standard deviations of the estimators.

\begin{tabular}{|c|c|c|c|c|c|c|c|c|}
\hline & \multicolumn{2}{|c|}{$H=0.65$} & \multicolumn{2}{|c|}{$H=0.70$} & \multicolumn{2}{|c|}{$H=0.75$} & \multicolumn{2}{|c|}{$H=0.85$} \\
\hline & $\alpha$ & $\beta$ & $\alpha$ & $\beta$ & $\alpha$ & $\beta$ & $\alpha$ & $\beta$ \\
\hline \multicolumn{9}{|c|}{ Panel A. Low parameter value $\alpha=0.7880, \beta=0.8215$} \\
\hline Mean & 0.7859 & 0.8166 & 0.7861 & 0.8280 & 0.7865 & 0.8164 & 0.7858 & 0.8333 \\
\hline Median & 0.7868 & 0.8120 & 0.7865 & 0.8348 & 0.7865 & 0.8115 & 0.7860 & 0.8452 \\
\hline Standard deviation & 0.1560 & 0.1030 & 0.1234 & 0.2933 & 0.7240 & 0.5533 & 0.8203 & 0.7709 \\
\hline \multicolumn{9}{|c|}{ Panel B. Median parameter value $\alpha=1.5880, \beta=1.8215$} \\
\hline Mean & 1.5845 & 1.8138 & 1.5832 & 1.8132 & 1.5851 & 1.8233 & 1.5829 & 1.8344 \\
\hline Median & 1.5863 & 1.8067 & 1.5839 & 1.8053 & 1.5849 & 1.8251 & 1.5837 & 1.8477 \\
\hline Standard deviation & 0.7819 & 0.5739 & 0.3454 & 0.2733 & 0.1074 & 0.5993 & 0.3145 & 0.3164 \\
\hline \multicolumn{9}{|c|}{ Panel C. High parameter value $\alpha=3.5880, \beta=5.8215$} \\
\hline Mean & 3.6069 & 5.9416 & 3.5999 & 5.7968 & 3.6031 & 5.9822 & 3.5741 & 5.7902 \\
\hline Median & 3.6215 & 6.0701 & 3.6002 & 5.7722 & 3.6056 & 6.1446 & 3.5782 & 5.7618 \\
\hline Standard deviation & 0.4331 & 0.4931 & 0.2272 & 0.2529 & 0.5654 & 0.7608 & 0.7594 & 1.0289 \\
\hline
\end{tabular}

estimators. We sum up the estimation procedures by Monte Carlo simulation method as follows.

(a) Based on Paxson [27] method, we generate fractional Gaussian noise by fast Fourier transform. Then using the result that a fractional Brownian motion is defined as the partial sums of the fractional Gaussian noise, we get the fractional Brownian motion.

(b) We simulate the processes $Y(\cdot)$ of (4) and $X(\cdot)$ of (5), using Euler-Mauyama method for different values of $H, \alpha$, and $\beta$. For simplicity, we set the valuation of the two variables $\alpha$ and $\beta$ according to the simulation part in Xiao et al. [17]. For a fixed length $\Delta t=0.01$, we simulate 100 sample paths on the interval $[0,20]$ using a regular partition of 2000 intervals.

(c) Calculate the term $T$. Here, we take $\varepsilon=0.009$ (in order to make sure that $\varepsilon=0.009$ is sufficiently small) as an example to show how to calculate $T$ in Assumption 5. If $H=0.85$, from the formula $T=$ $(\varepsilon / K)^{1 /(2 H-4)}$, we need to choose $T=13.39$. Hence, for the estimation procedure, we only use $\left\{W_{k, N}\right\}, k=$ $13391, \ldots, 20000$.

(d) We implement these generated data sets to obtain the estimators by (25).

The simulated mean, median value, and standard deviation of these estimators are given in Table 1. As shown in Table 1, both mean and median value of all parameters are close to the true value. The standard deviations are small which also demonstrated a good finite sample behavior of our method. There is not a decrease in quality as $H$ increases. The theoretical results in Section 2 are confirmed by these simulation studies. In summary, the simulation results match the chosen parameters exactly, which shows that our estimators perform well.

Finally, in order to illustrate the asymptotical normality for the estimators, we plot the histogram of the empirical distribution that we obtain from the Monte Carlo procedure (see Figures 1, 2, 3, 4, 5, and 6).

\section{Conclusion}

The process of long memory has evolved into the description of stochastic volatility during the last decades, as researchers in empirical finance have sought to use longmemory stochastic volatility models in terms of discrete time and continuous time for practical application. Among them, fractional Ornstein-Uhlenbeck process has been used by many authors for its brief form and good properties. However, no volatility process can be directly observed from the financial market. We get the approximate logarithm form of $\left\{e^{Y_{i}} \xi_{i}\right\}$ in the discrete time, which is available for us to adopt the least squares variogram method. For this reason, obtaining the estimators $\alpha$ and $\mu$ would be feasible. This paper establishes an ordinary least squares estimation of the variogram parameters to deal with the case where the volatility process is described by a fractional OrnsteinUhlenbeck process. We also give the results for the asymptotic properties of the two estimators. The numerical results show that this is an accurate and reliable method to estimate the parameters.

As stated in this paper, we assume the mutual independence between $\left\{W_{t}\right\}$ and $\left\{B_{t}^{H}\right\}$ for showing the estimation procedure clearly. In order to consider leverage effects, we would need to impose some kind of dependent structure on the covariance matrix of these two processes. Such dependent structure would make the current discussion much more complicated and we thus wish to leave such an extension for future research.

\section{Appendix}

Proof of Lemma 1. Note that the $\mathrm{fBm}\left\{B_{t}^{H}\right\}$ is a Gaussian process with $E\left[B_{t}^{H}\right]=0$ for every $t \in \mathbb{R}$. Hence, we can easily 


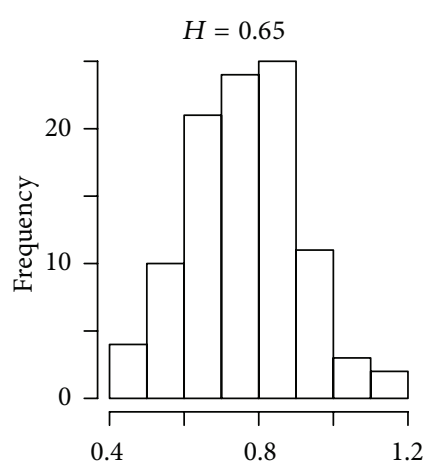

(a)

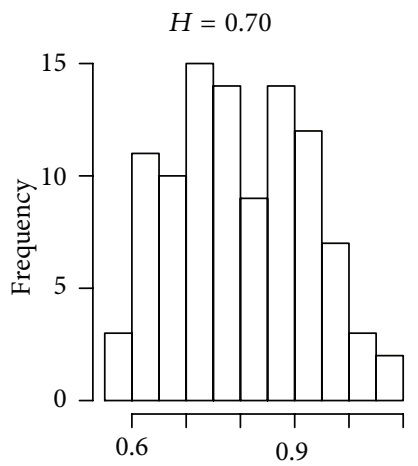

(b)

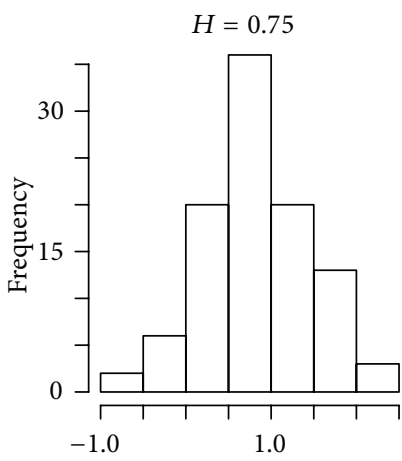

(c)

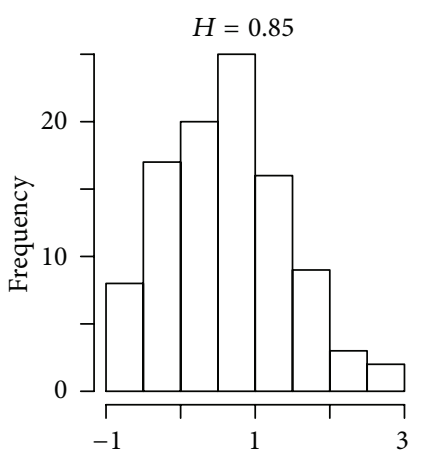

(d)

FIGURE 1: Histogram of the estimator $\widehat{\alpha}(\alpha=0.7880)$ for different values of $H$.

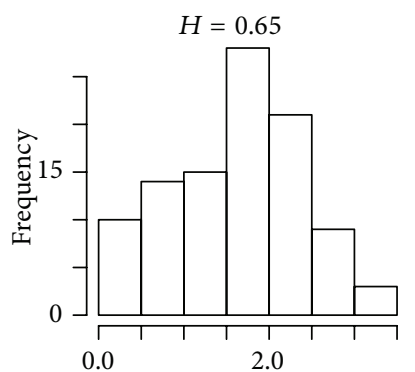

(a)

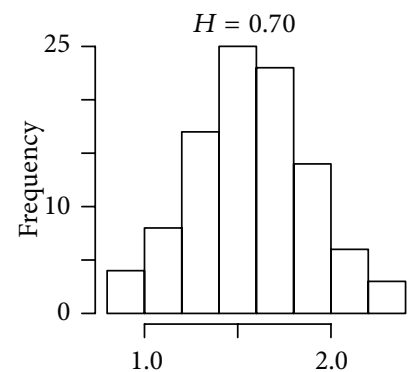

(b)

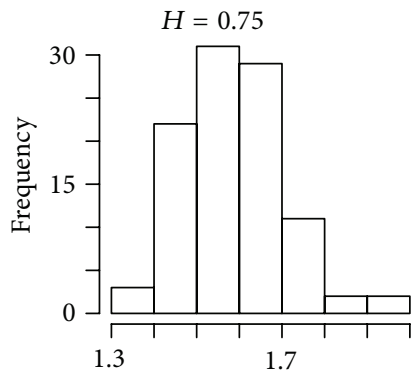

(c)

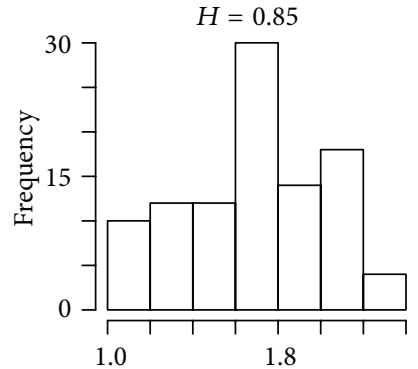

(d)

FIgURE 2: Histogram of the estimator $\widehat{\alpha}(\alpha=1.5880)$ for different values of $H$.

obtain that $E\left[Y_{t}\right]=0$ and $\left\{Y_{t}\right\}$ is also a Gaussian process. Based on Theorem 3.1 in Nualart [29], the variance of $\left\{Y_{t}\right\}$ is

$$
\begin{aligned}
& \operatorname{Var}\left[Y_{t}\right] \\
& =E\left[\beta \int_{-\infty}^{t} e^{-\alpha(t-r)} d B_{r}^{H} \beta \int_{-\infty}^{t} e^{-\alpha(t-u)} d B_{u}^{H}\right] \\
& =H(2 H-1) \beta^{2} e^{-2 \alpha t} \\
& \quad \times\left[\int_{-\infty}^{t} e^{\alpha r} \int_{-\infty}^{r}(r-u)^{2 H-2} e^{\alpha u} d u d r\right. \\
& \left.\quad+\int_{-\infty}^{t} e^{\alpha r} \int_{r}^{t}(u-r)^{2 H-2} e^{\alpha u} d u d r\right] .
\end{aligned}
$$

Set

$$
\begin{gathered}
\mathrm{I}=e^{-2 \alpha t} \int_{-\infty}^{t} e^{\alpha r} \int_{-\infty}^{r}(r-u)^{2 H-2} e^{\alpha u} d u d r, \\
\mathrm{II}=e^{-2 \alpha t} \int_{-\infty}^{t} e^{\alpha r} \int_{r}^{t}(u-r)^{2 H-2} e^{\alpha u} d u d r .
\end{gathered}
$$

Let $u-r=v, \alpha v=x$. Similarly, we get that

$$
\begin{aligned}
\mathrm{II} & =e^{-2 \alpha t} \int_{-\infty}^{t} e^{\alpha r} \int_{r}^{t}(u-r)^{2 H-2} e^{\alpha u} d u d r \\
& =e^{-2 \alpha t} \int_{0}^{\infty} v^{2 H-2} e^{\alpha \nu} \int_{-\infty}^{t-v} e^{2 \alpha r} d r d v \\
& =\frac{1}{2} \alpha^{-2 H} \int_{0}^{\infty} x^{2 H-2} e^{-x} d x \\
& =\frac{1}{2} \alpha^{-2 H} \Gamma(2 H-1) .
\end{aligned}
$$




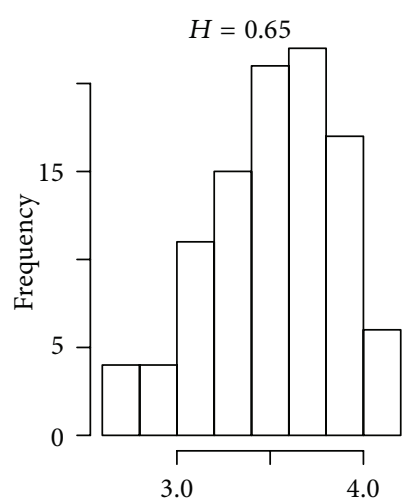

(a)

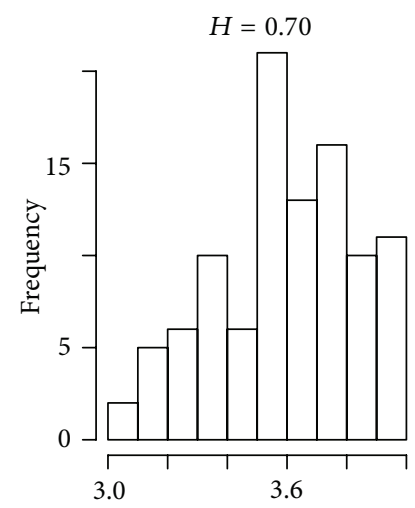

(b)

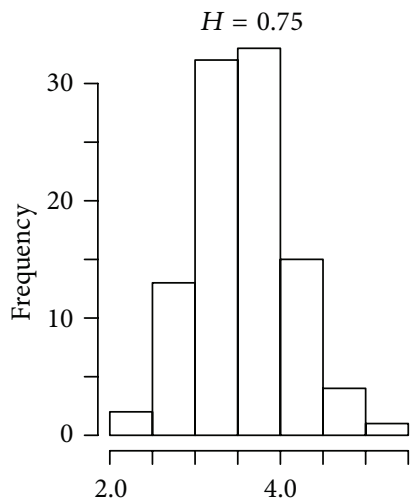

(c)

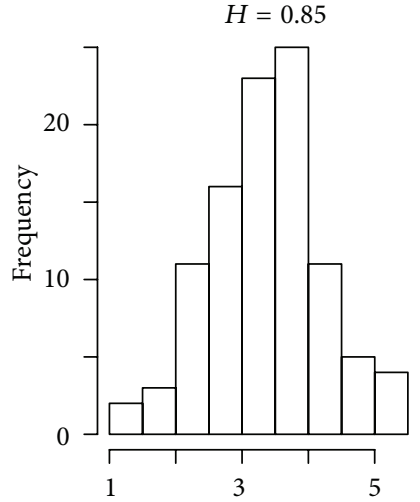

(d)

FIGURE 3: Histogram of the estimator $\widehat{\alpha}(\alpha=3.5880)$ for different values of $H$.

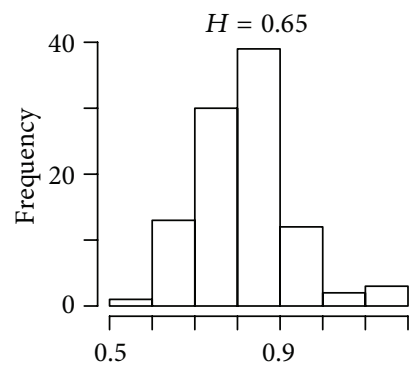

(a)

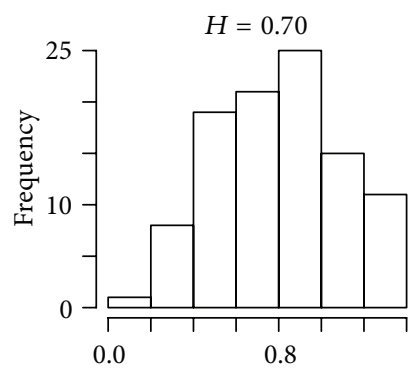

(b)

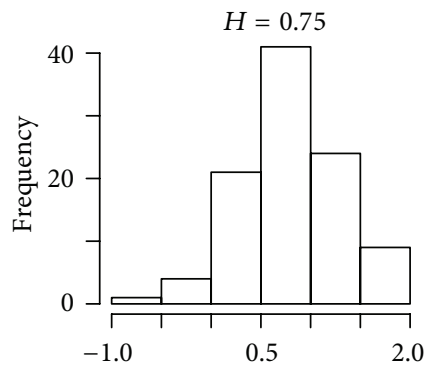

(c)

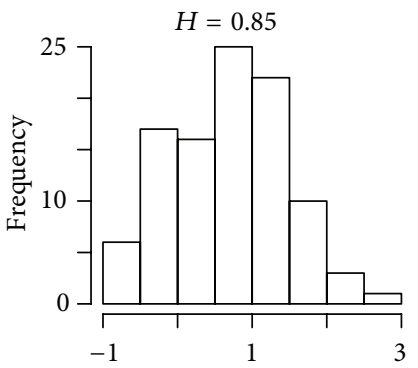

(d)

FIgURE 4: Histogram of the estimator $\widehat{\beta}(\beta=0.8215)$ for different values of $H$.

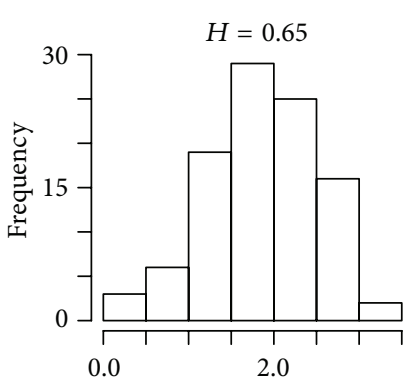

(a)

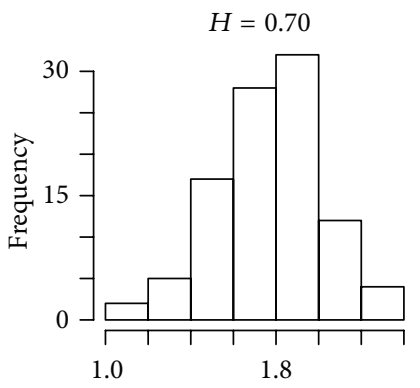

(b)

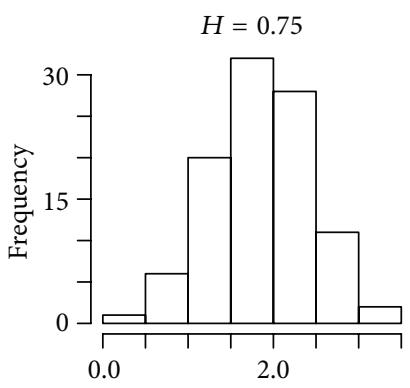

(c)

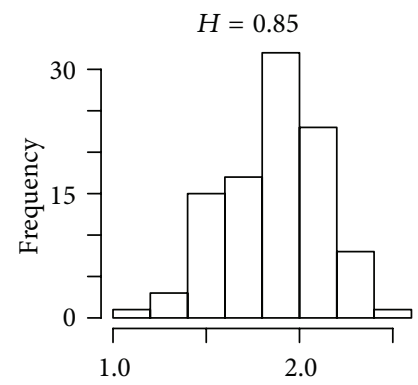

(d)

Figure 5: Histogram of the estimator $\widehat{\beta}(\beta=1.8215)$ for different values of $H$.

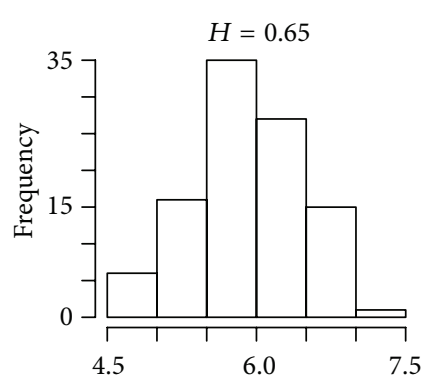

(a)

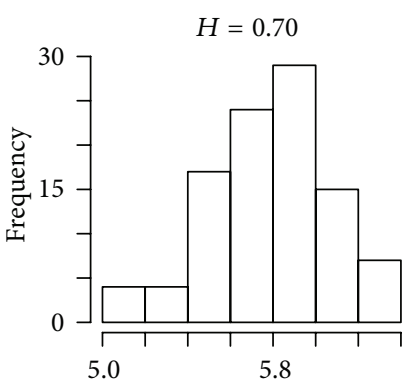

(b)

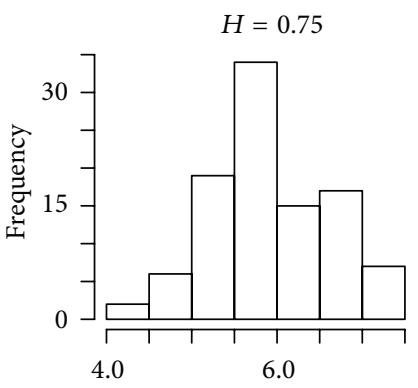

(c)

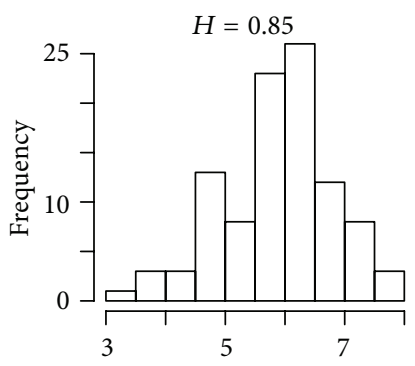

(d)

FIgURE 6: Histogram of the estimator $\widehat{\beta}(\beta=5.8215)$ for different values of $H$. 
Hence, $\operatorname{Var}\left[Y_{t}\right]$ can be written as

$$
\begin{aligned}
\operatorname{Var}\left[Y_{t}\right] & =H(2 H-1) \beta^{2} \alpha^{-2 H} \Gamma(2 H-1) \\
& =\Gamma(2 H) H \alpha^{-2 H} \beta^{2}
\end{aligned}
$$

Thus, we deduce the desired conclusion.

\section{Conflict of Interests}

The authors declare that there is no conflict of interests regarding the publication of this paper.

\section{Acknowledgments}

The authors are very grateful to many anonymous reviewers for their insightful comments and suggestions. This research is supported by the National Social Science Foundation of China (no. 11\&ZD156).

\section{References}

[1] S. J. Taylor, Modeling Financial Time Series, John Wiley \& Sons, Chichester, UK, 1986.

[2] P. M. Robinson, Time Series with Long Memory, Advanced Texts in Econometrics, Oxford University Press, Oxford, UK, 2003.

[3] F. J. Breidt, N. Crato, and P. de Lima, "The detection and estimation of long memory in stochastic volatility," Journal of Econometrics, vol. 83, no. 1-2, pp. 325-348, 1998.

[4] A. C. Harvey, "Long memory in stochastic volatility," in Forecasting Volatility in Financial Markets, pp. 307-320, Butterworth-Haineman, Oxford, UK, 2002.

[5] P. M. Robinson and P. Zaffaroni, "Nonlinear time series with long memory: a model for stochastic volatility," Journal of Statistical Planning and Inference, vol. 68, no. 2, pp. 359-371, 1998.

[6] F. Comte and E. Renault, "Long memory in continuous-time stochastic volatility models," Mathematical Finance, vol. 8, no. 4, pp. 291-323, 1998.

[7] F. Comte, L. Coutin, and E. Renault, "Affine fractional stochastic volatility models," Annals of Finance, vol. 8, no. 2-3, pp. 337-378, 2012.

[8] A. Chronopoulou and F. G. Viens, "Estimation and pricing under long-memory stochastic volatility," Annals of Finance, vol. 8, no. 2-3, pp. 379-403, 2012.

[9] J. Geweke and S. Porter-Hudak, "The estimation and application of long memory time series models," Journal of Time Series Analysis, vol. 4, no. 4, pp. 221-238, 1983.

[10] R. S. Deo and C. M. Hurvich, "On the log periodogram regression estimator of the memory parameter in long memory stochastic volatility models," Econometric Theory, vol. 17, no. 4, pp. 686-710, 2001.

[11] J. Arteche, "Gaussian semiparametric estimation in long memory in stochastic volatility and signal plus noise models," Journal of Econometrics, vol. 119, no. 1, pp. 131-154, 2004.

[12] W. W. Chen and R. S. Deo, "Power transformations to induce normality and their applications," Journal of the Royal Statistical Society. Series B. Statistical Methodology, vol. 66, no. 1, pp. 117130, 2004
[13] P. J. Brockwell and R. A. Davis, Time Series: Theory and Methods, Springer Series in Statistics, Springer, New York, NY, USA, 2nd edition, 2009.

[14] I. Casas and J. Gao, "Econometric estimation in long-range dependent volatility models: theory and practice," Journal of Econometrics, vol. 147, no. 1, pp. 72-83, 2008.

[15] A. Chronopoulou and F. G. Viens, "Stochastic volatility and option pricing with long-memory in discrete and continuous time," Quantitative Finance, vol. 12, no. 4, pp. 635-649, 2012.

[16] P. Cheridito, H. Kawaguchi, and M. Maejima, "Fractional Ornstein-Uhlenbeck processes," Electronic Journal of Probability, vol. 8, no. 3, pp. 1-14, 2003.

[17] W. Xiao, W. Zhang, and W. Xu, "Parameter estimation for fractional Ornstein-Uhlenbeck processes at discrete observation," Applied Mathematical Modelling, vol. 35, no. 9, pp. 4196-4207, 2011.

[18] Y. Hu and D. Nualart, "Parameter estimation for fractional Ornstein-Uhlenbeck processes," Statistics \& Probability Letters, vol. 80, no. 11-12, pp. 1030-1038, 2010.

[19] M. Rosenbaum, "Estimation of the volatility persistence in a discretely observed diffusion model," Stochastic Processes and Their Applications, vol. 118, no. 8, pp. 1434-1462, 2008.

[20] F. G. Viens, "Portfolio optimization under partially observed stochastic volatility," in Proceedings of the 8th International Conference on Advances in Communication and Control (COMCON '02), W. Wells, Ed., pp. 1-12, Optimization Software, Inc., 2002.

[21] L. Scott, "Option pricing when the variance changes randomly: theory, estimation, and an application," Journal of Financial and Quantitative Analysis, vol. 22, no. 3, pp. 419-438, 1987.

[22] I. Nourdin, "Schémas d'approximation associés à une équation différentielle dirigée par une fonction höldérienne; cas du mouvement brownien fractionnaire," Comptes Rendus de l'Académie des Sciences. Series I. Mathematics, vol. 340, no. 8, pp. 611-614, 2005.

[23] A. Neuenkirch and I. Nourdin, "Exact rate of convergence of some approximation schemes associated to SDEs driven by a fractional Brownian motion," Journal of Theoretical Probability, vol. 20, no. 4, pp. 871-899, 2007.

[24] Y. S. Mishura, Stochastic Calculus for Fractional Brownian Motion and Related Processes, vol. 1929 of Lecture Notes in Mathematics, Springer, Berlin, Germany, 2008.

[25] R. Belfadli, K. Es-Sebaiy, and Y. Ouknine, "Parameter estimation for fractionalOrnstein-Uhlenbeck processes: non-ergodic case," http://arxiv.org/abs/1102.5491.

[26] S. N. Lahiri, Y. Lee, and N. Cressie, “On asymptotic distribution and asymptotic efficiency of least squares estimators of spatial variogram parameters," Journal of Statistical Planning and Inference, vol. 103, no. 1-2, pp. 65-85, 2002.

[27] V. Paxson, "Fast approximate synthesis of fractional Gaussian noise for generating self-similar network traffic," Computer Communication Review, vol. 27, no. 5, pp. 5-18, 1997.

[28] A. R. Hall, Generalized Method of Moments, Advanced Texts in Econometrics, Oxford University Press, Oxford, UK, 2005.

[29] D. Nualart, "Fractional Brownian motion: stochastic calculus and applications," in International Congress of Mathematicians. Vol. III, pp. 1541-1562, European Mathematical Society, Zürich, Switzerland, 2006. 


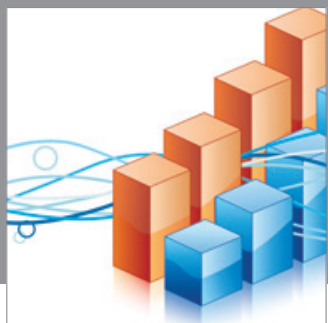

Advances in

Operations Research

mansans

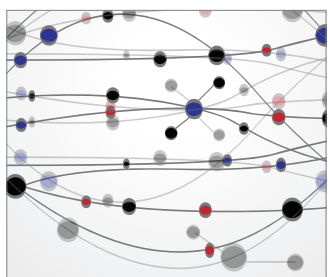

The Scientific World Journal
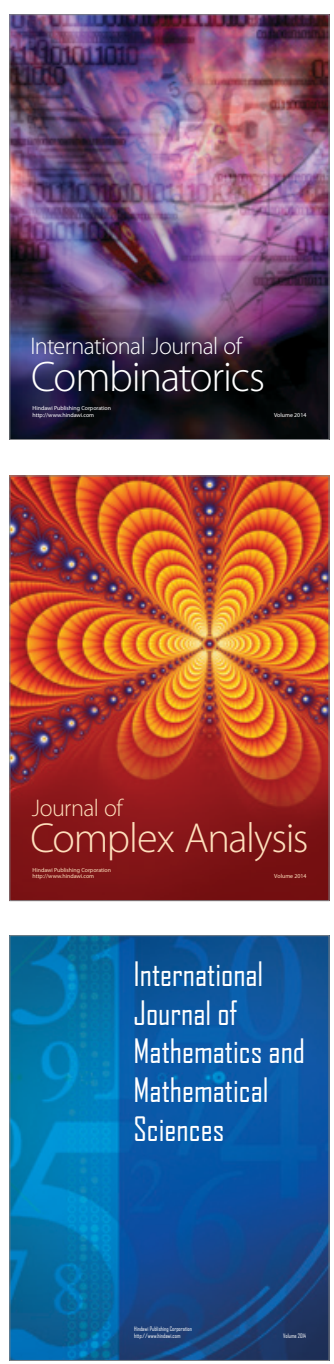
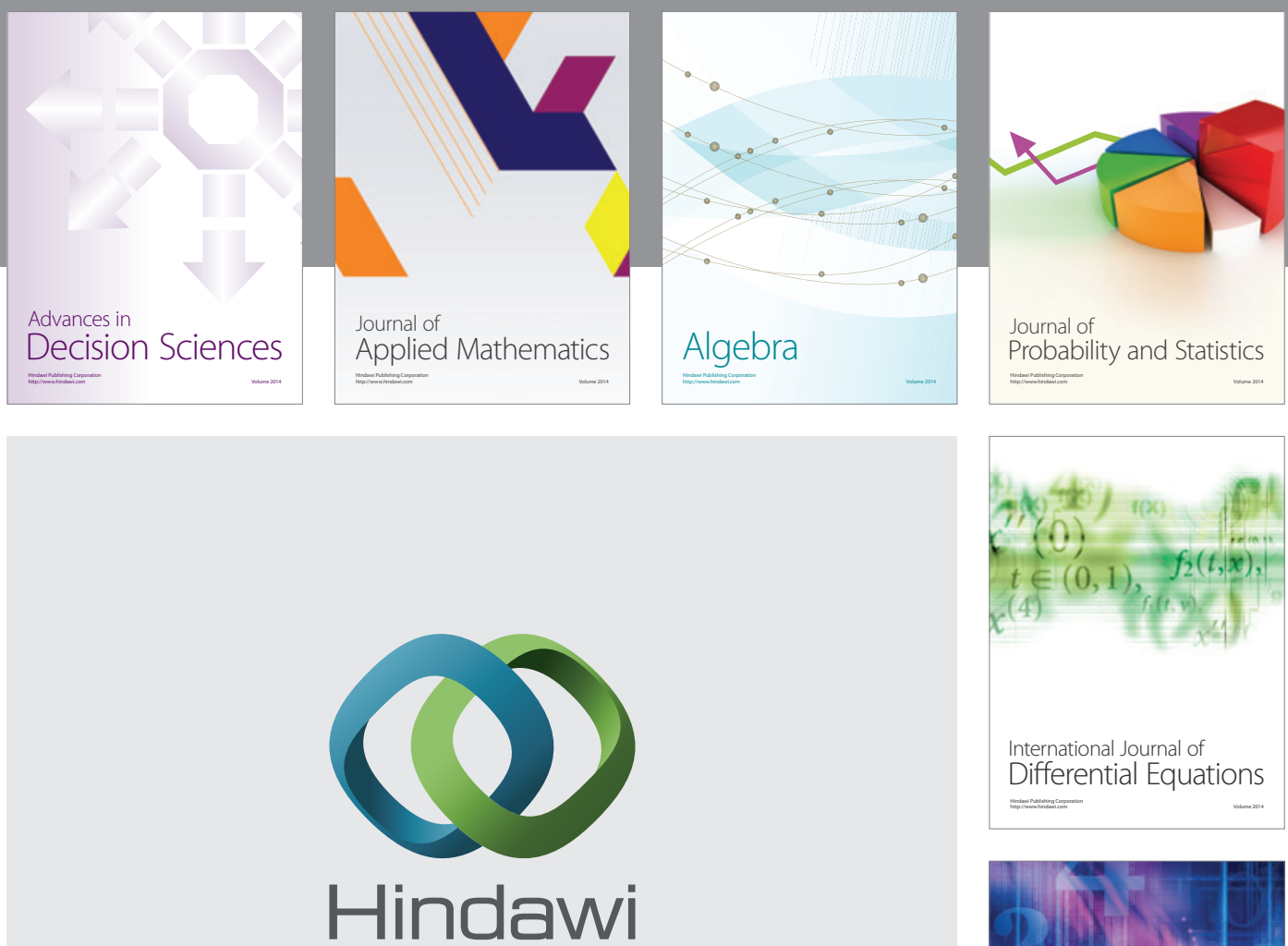

Submit your manuscripts at http://www.hindawi.com
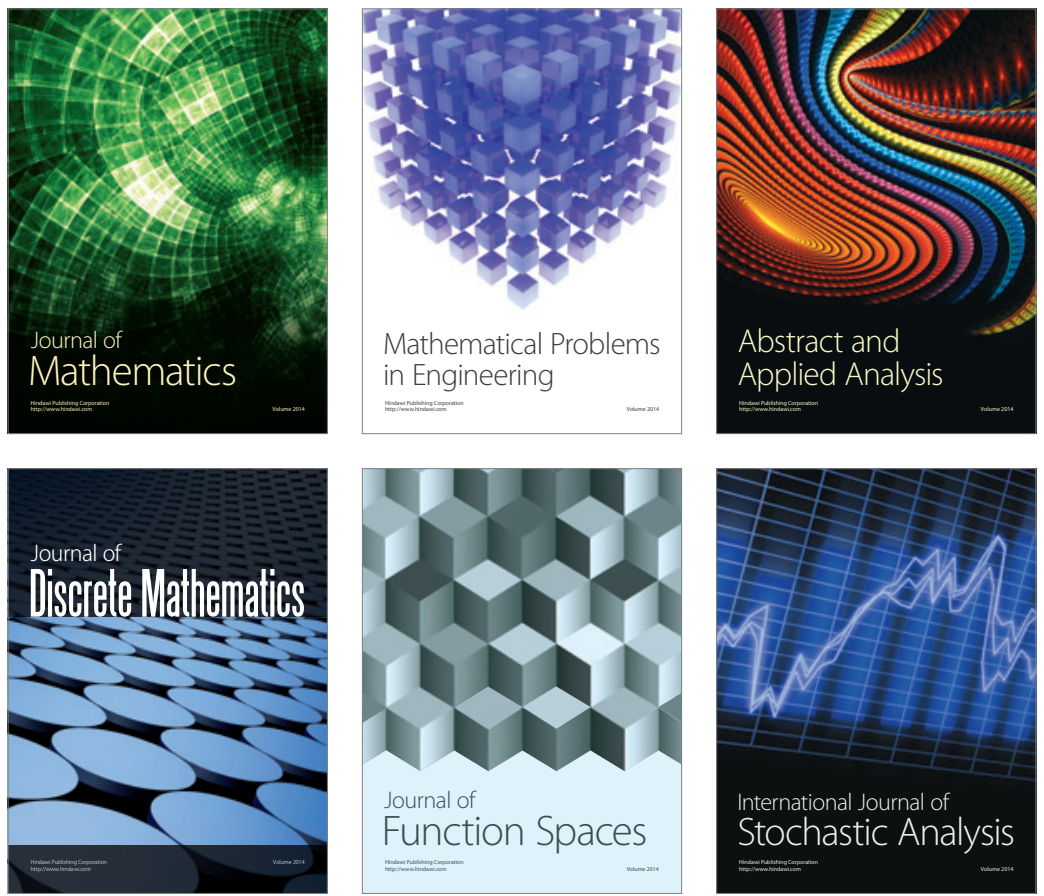

Journal of

Function Spaces

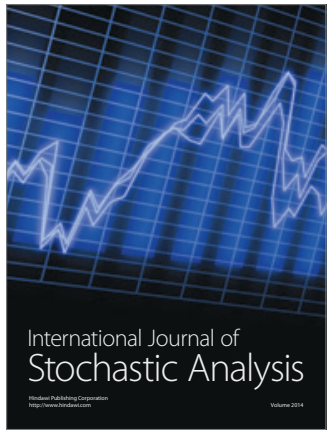

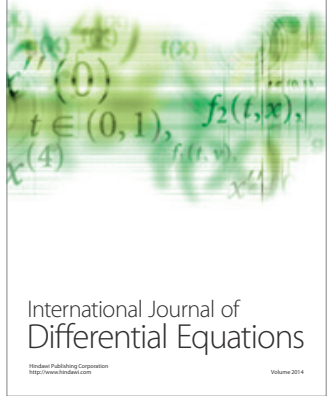
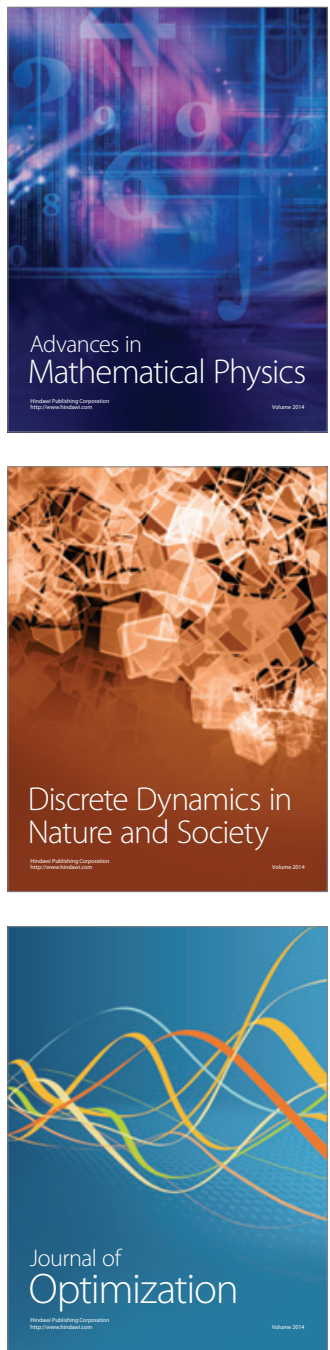